\title{
Endodontic Periodontic Lesions and Host Modulation - Case Series
}

\author{
Afaf Zia ${ }^{1}$, Afshan Bey ${ }^{1}$, Syed MUN. Andrabi², Saif Khan ${ }^{1}$, Masood Khan ${ }^{3}$ \\ ${ }^{1}$ Department of Periodontology, Dr ZA Dental College, Aligarh Muslim University, Aligarh 202001, India \\ ${ }^{2}$ Department of Conservative and Endodontics, Aligarh Muslim University, Aligarh 202001, India \\ ${ }^{3}$ Department of Oral Diagnosis and Oral Pathology, Aligarh Muslim University, Aligarh 202001, India \\ Correspondence e-mail to: afafzia@gmail.com
}

\begin{abstract}
Host modulation, includes inhibition of matrix metalloproteinases, blocking production of proinflammatory cytokines and inhibition of osteoclastic activity, has therapeutic value as adjunctive therapy in treating chronic periodontitis. This case series presented three patients with chief complaint of teeth mobility where endo perio involvement was diagnosed. The endo perio combined lesions generally shows the communication between pulp and periodontal tissues with the same origin of dental infection. Diagnosis in such cases is difficult and generally raising problem in the management. All of the cases were managed by non surgical endodontic therapy combined with host modulation. All local factors causing the lesion were removed. Clinical outcome after six and nine months were quite satisfactory. Combined endo perio cases are challenging to the dentists and involve multidisciplinary involvement. To manage such cases, dentists have to rely on the unconventional techniques.
\end{abstract}

Key words: endo perio lesion, host modulation, non surgical therapy

\section{INTRODUCTION}

Combined endo perio lesions are frequently encountered in dental clinics and are difficult to manage. Non surgical therapy like scaling, root planing and root canal therapy is the preferred choice of treatment. At times, surgical therapy like open flap debridement and regenerative techniques may be required. To overcome the need of surgery, a new therapy of host modulation is used nowadays. Host modulation is a recent concept where the proinflammatory mediators and destructive enzymes in the host are modified. Though for the initiation of any periodontal diseases pathogens are required, they themselves cannot cause periodontal disease. A susceptible host is needed. Hence the agents that can modify host responses so as to restore the balance of proinflammatory or destructive mediators and anti inflammatory or protective mediators could play a significant role in treatment of periodontal diseases. ${ }^{1}$ Over the last two decades, a variety of pharmacological agents have been studied for a possible role as host modulators in the management of periodontal disease. These include subantimicrobial dose doxycycline, non-steroidal anti inflammatory drugs, bisphosphonates and many other newer drugs. In the Host modulation therapy the balance between health and disease progression is directed towards healing response. This therapy when used in combination with conventional therapies like scaling and root planning render clinical improvement and reversal of bone loss is seen. ${ }^{2}$ We report here three combined endo perio leions where host modulation therapy has been used along with the conventional technique. 


\section{CASE REPORT}

\section{CASE 1}

A 25 year old female patient reported with slight pain and extreme mobility in right lower back teeth since 3 months. The patient was systemically healthy and there was no medical history contributory to the dental problem. Clinically, on probing teeth 44 , a pocket around $11 \mathrm{~mm}$ was seen on both mesial and distal sides. Grade II mobility was present. Tooth was vital and the pain may be due to secondary endodontic involvement. Plaque and calculus deposits were present and generalised pockets were also seen. X-ray revealed periapical radiolucency and excessive bone loss in relation to tooth 44. A diagnosis of chronic periodontitis was made. In tooth 44 there was probably secondary endodontic involvement (Figure 1a).

Treatment involved initially removal of all deposits by thorough scaling and root planning. Host modulation therapy was advocated from the day diagnosis was made that is the patient was placed on subantimicrobial dose doxycycline for 9 months twice daily. Subantimicrobial dose doxycycline has been our choice of drug as it is approved in most countries for host modulation. ${ }^{6}$ No side effects were observed following its administration. Three weeks after scaling and root planning, root canal therapy was done on all relevant teeth. At every recall visit oral hygiene instructions was reinforced. Follow up radiographs were taken at 6 months, 9 months and 1 year.

The patient maintained hygiene and the relevant involved teeth showed reduced mobility and patient was able to chew properly. After 6 months, there was apparent bone fill figure $1 \mathrm{~b}$. After 9 months radiographically, there was bone fill (Figure 1c) and after a year marked bone fill was seen (Figure 1d).

\section{CASE 2}

A 45 year old female came with the chief complaint of excessive mobility and slight pain in relation to lower front teeth. The patient had no contributory medical history to dental problem. Clinically, 31 and 42 teeth were highly mobile and recession was seen. There was excessive plaque and calculus deposits and trauma from occlusion in the lower anterior incisors. Tooth 41 was extracted for periodontal reasons. Radiographically there was generalised bone loss. Teeth 31 and 42 showed immense bone loss and there was pain on percussion (Figure $2 \mathrm{a}$ ). This pain may be due to either trauma from occlusion or secondary endodontic involvement. Patient was diagonosed with chronic periodontitis.

Patient was managed in similar way as the previous case. Scaling and root planning was done and placed on host modulation therapy similar to the previous case. Trauma was removed in the lower anteriors by selective grinding. Root canal therapy was done in teeth 31 and 42 after three months. Radiographs after 6 and 9 months showed slight bone fill figure $2 \mathrm{~b}$ and $\mathrm{c}$ and marked bone fill was seen after a year (Figure 2d). On surgical entry after one year complete bone formation was seen.

\section{CASE 3}

A 24 Year old female came with chief complaint of mobility and slight pain in upper front teeth. Clinically, teeth 22 and 23 showed slight mobility and there was a pocket of $6 \mathrm{~mm}$ around 22 and 23. Plaque and calculus deposits were seen. On palpation and percussion there was slight pain which may be due to secondary pulpal involvement. Radiograph showed vertical bone resorption in relation to 23 (Figure 3a). Diagnosis of chronic periodontitis was made with secondarily endodontic involvement in 22 and 23.

Treatment rendered was similar to above mentioned cases. Scaling and root planning was done and placed on host modulation similar to the above mentioned cases. Thereby, root canal therapy was done after three months. Radiographs showed bone fill after 9 months and one year (Figure 3b dan c).

\section{DISCUSSION}

The endo-perio lesion is a condition characterized by presence of endodontic and periodontal disease because of their common dental origin. The relationship between these two diseases was first described by Goldberg in 1964. ${ }^{3}$ The deletorius effect of pulp on periodontium is well documented. The pulp can be invaded from periodontium through apical foramen, accessory canals dan lateral canals. ${ }^{4,5}$ Combined endodontic-periodontic lesions present a great dilemma to the dentist as to what should be the approach of treatment. Such cases are generally managed by both non surgical and surgical phase. Non surgical phase includes thorough scaling and root planing and root canal treatment after a period of three months. Surgical phase involves better cleaning of root surfaces and filling of the advanced resorbed defects with grafts and membranes.

This present study reports three cases of primarily periodontal involvement with secondary endodontic involvement and their management. We have in our cases used the non surgical therapy. Instead of surgically opening the defects and filling with bone fill material, we have utilised the concept of host modulation. Periodontal diseases are initiated in presence of periodontopathic micro-organisms and a susceptible host. Therefore even if the pathogenic bacteria are present but the host derived destructive enzymes and mediators are not activated, the disease process wouldn't be initiated. Host modulation means modifying or modulating destructive or damaging aspects of the inflammatory host response that develops in the periodontal tissues as a result of the chronic challenge presented by the subgingival bacterial 

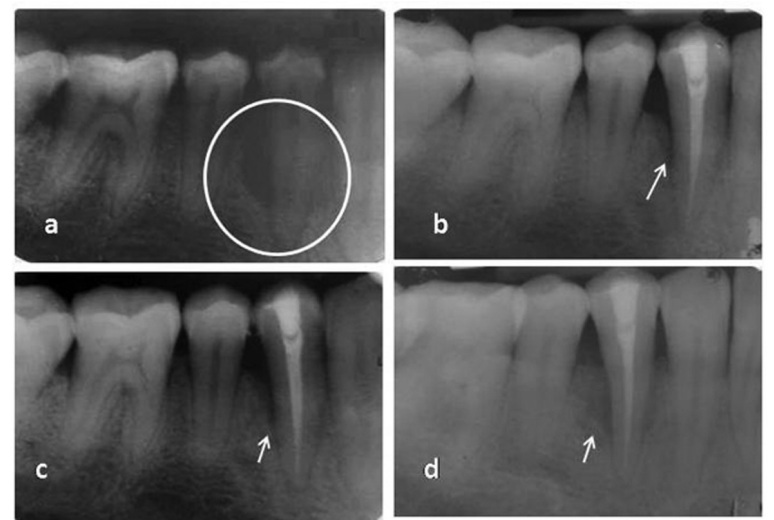

Figure 1. Case 1 IOPA radiograph showing endoperio lesion encircled (a), follow up -6 months (b), follow up -9 months (c), follow up -1 year (d). Arrow indicates areas of bone fill.
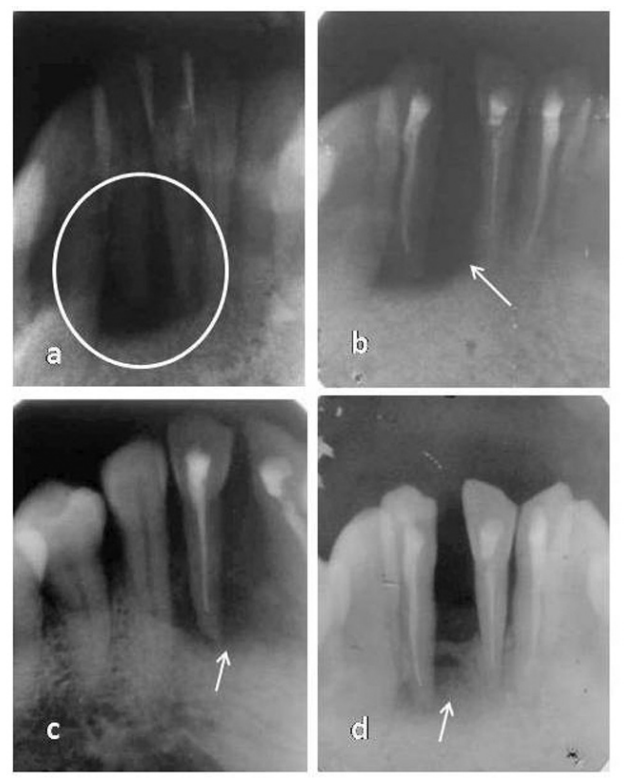

Figure 2. Case 2 radiograph showing endoperio lesion encircled (a), follow up -6 months (b), follow up -9 months (c), follow up -1 year (d). Arrow indicates areas of bone fill.

plaque. ${ }^{6}$ The rationale behind the use of this therapy is that it helps the host in fighting against the infectious agents by modifying the host responses to inflammatory mediators. ${ }^{9}$ This host modulation therapy has fewer side effects, is non-invasive and does not require difficult application methods. Another advantage is that it can be initiated as an adjunct therapy at any age. In certain situations conventional therapy may not result in the desired results. In such cases if one additionally modulates the host response, effective results can be gained. Hence, we have started host modulation therapy in all three cases from the beginning of the treatment as an adjunct to conventional therapy. Various host modulating therapies have been proposed which block the pathways of tissue destruction. This includes subantimicrobial dose of doxycycline, non steroidal anti inflammatory drugs and bisphosphonates ${ }^{10}$. The
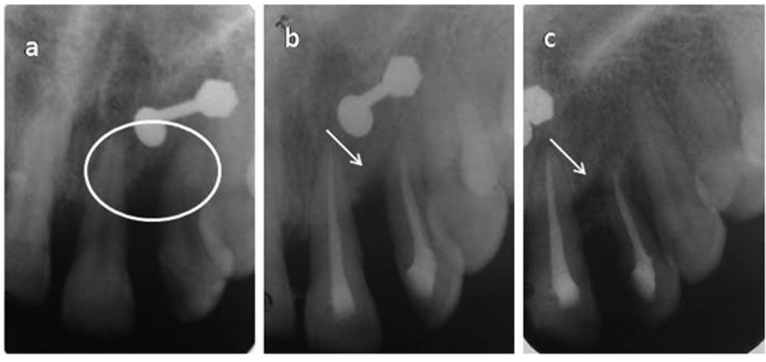

Figure 3. Case 3 IOPA radiograph showing endoperio lesion encircled (a), follow up - 9 months (b), follow up - 1 year $(c)$. Arrow indicates areas of bone fill.

newer approaches are lipoxins, resolvins, nitric oxide synthase activity inhibitor and chemically modified tetracyclines ${ }^{11}$. Subantimicrobial dose doxycycline is approved by the US Food and Drug Administration, the UK Medicines and Healthcare products Regulatory Agency and by similar agencies in other countries throughout the world ${ }^{6}$ and hence was the drug of choice for host modulation in all our cases.

In all our cases, there was primarily periodontal problem associated with secondary endodontic involvement. After scaling and root planing in all cases, there was slight decrease in mobility of teeth. This scaling and root planning is considered as gold standard for treatment for any periodontal lesion as it reduces the chronic challenge presented by the subgingival bacteria. This results in reduction of inflammatory responses in the periodontal tissue and clinically as well there is resolution of inflammation. In case 2 , mobility was reduced immensely after removing the trauma. Patients were placed on maintainence therapy to evaluate their efficacy in maintaining oral hygiene. Root canal therapy was done after three months. Radiograph after 6 months, showed slight bone fill in all the defects around the root canal treated teeth. Radiograph after 9 months showed immense bone fill. This regeneration may be due to combined effect of root canal therapy and host modulation. After 12 months, in all cases bone regeneration was to a greater extent. This bone formation may be due to decreased inflammation as a result of decreased microbial load by scaling and root planing. Also root canal therapy prevents the spread of inflammatory mediators from the pocket. Doxycycline as a host modulating agent inhibits host connective tissue breakdown through different mechanisms. Doxycycline at subantimicrobial doses inhibits the activity of matrixmetalloproteinases (MMPs), which are capable of degrading various extracellular molecules including collagen. ${ }^{7}$ In the connective tissue, doxycycline reduces osteoclast activity and bone resorption, blocks osteoclast MMP and stimulates osteoblasts activity and bone formation. ${ }^{12}$ Doxycycline 
potentiates osseous regeneration in periodontal defects due to its anti-collagenase activity. ${ }^{13}$ Subantimicrobial dose doxycycline has the advantage of being safe with minimal side effects because of low plasma concentration (peak concentrations of $0.7-0.8 \mu \mathrm{g} / \mathrm{ml}$ and steady- state concentrations of approximately $0.4 \mu \mathrm{g} / \mathrm{ml}){ }^{8,14}$ While the concentrations of $3-4 \mu \mathrm{g} / \mathrm{ml}$ is expected following antibiotic doses of $100-200 \mathrm{mg}$ of doxycycline and thereby adverse effects are observed. ${ }^{8}$ No evidence of antibiotic resistance has been identified in any of the studies that have investigated this issue till date. To our knowledge, this is one of the first case reports where endo perio lesions are managed by host modulation.

\section{CONCLUSION}

In light of the treatment responses observed in this study, the healing potential of periodontal tissue, loss due to severe periodontitis treated with non-surgical and adjunctive therapy for host modulation requires further investigation. The host modulation appears to be an impetus for impressive clinical responses to non-surgical therapy. There is little information available on many host modulating therapies related to periodontitis and scarce data on the application of the host modulation therapy. Future developments in relation to subantimicrobial dose doxycycline will include modified-release formulations that achieve sustained plasma concentrations of doxycycline over 24 hours, but only require once per day dosing, thereby improving patient compliance. Thus, they should form part of an integrated treatment approach, together with hygiene therapy, plaque control, root surface instrumentation, maintenance care and risk factor modification.

\section{REFERENCES}

1. Elavarasu S, Sekar S, Murugan T. Host modulation by therapeutic agents. J Pharm Bioallied Sci. 2012;4:75-9.

2. Caton JG, Ciancio SG, Blieden TM, Bradshaw M, Crout RJ, et al. Treatment with subantimicrobial dose doxycycline improves the efficacy of scaling and root planing in patients with adult periodontitis. J Periodontol. 2000;71:521-32.

3. Simring M, Goldberg M. The pulpal pocket approach: retrograde periodontitis. J Periodontol. 1964;35: 22-48.

4. Adriaens PA, De Boever JA, Loesche WJ. Bacterial invasion in root cementum and radicular dentin of periodontally diseased teeth in humans. A reservoir of periodontopathic bacteria. J Periodontol. 1988;59:222-30.

5. Czarnecki RT, Schilder H. A histologic evaluation of the human pulp in teeth with varying degrees of periodontal disease. J. Endod. 1979;5:242-53.

6. Preshaw PM. Host response modulation in periodontics. Periodontology 2000. 2008:48:92110.

7. Birkedal-Hansen H. Role of matrix metalloproteinases in human periodontal diseases. J Periodontol. 1993;64:474-84.

8. Walker C, Thomas J, Nango S, Lennon J, Wetzel J, et al. Long-term treatment with subantimicrobial dose doxycycline exerts no antibacterial effect on the subgingival microflora associated with adult periodontitis. J Periodontol.2000;71:1465-71.

9. Salvi GE, Lang NP. Host response modulation in the management of periodontal diseases. J Clin Periodontol. 2005;32:108-29.

10. Reddy MS, Geurs NC, Gunsolley JC. Periodontal host modulation with antiproteinase, antiinflammatory, and bone-sparing agents. A systematic review. Ann Periodontol. 2003;74:12-37.

11. Bhatavadekar NB, Williams RC. Commentary: New directions in host modulation for the management of periodontal diseases. J Clin Periodontol. 2009;36:124-6.

12. Zia A, Bey A, Andrabi SMUN. Host modulation for management of periodontal diseases. Guident. 2014:4:53-6.

13. Golub LM, McNamara TF, Ryan ME, Kohut B, Blieden T. Adjunctive treatment with substantimicrobial doses of doxycycline: effects on gingival fluid collagenase activity and attachment loss in adult periodontitis. J Clin Periodontol. 2001;28:146-56.

14. Lee HM, Ciancio SG, Tüter G, Ryan ME, Komaroff E, Golub LM. Subantimicrobial dose doxycycline efficacy as a matrix metalloproteinase inhibitor in chronic periodontitis patients is enhanced when combined with a non-steroidal anti-inflammatory drug. J Periodontol. 2004;75:453-63.

(Received September 3, 2014; Accepted December 9, 2014) 